\title{
Imaging of an Early Memory Trace in the Drosophila Mushroom Body
}

\author{
Yalin Wang, ${ }^{1 \star}$ Akira Mamiya, ${ }^{1 \star}$ Ann-shyn Chiang, ${ }^{2}$ and Yi Zhong ${ }^{1}$ \\ ${ }^{1}$ Cold Spring Harbor Laboratory, Cold Spring Harbor, New York 11724, and 2 Institute of Biotechnology, National Tsing Hua University, Hsinchu 30043, \\ Taiwan, Republic of China
}

Extensive molecular, genetic, and anatomical analyses have suggested that olfactory memory is stored in the mushroom body (MB), a higher-order olfactory center in the insect brain. The MB comprises three subtypes of neurons with axons that extend into different lobes. A recent functional imaging study has revealed a long-term memory trace manifested as an increase in the $\mathrm{Ca}^{2+}$ activity in an axonal branch of a subtype of MB neurons. However, early memory traces in the MB remain elusive. We report here learning-induced changes in $\mathrm{Ca}^{2+}$ activities during early memory formation in a different subtype of MB neurons. We used three independent in vivo and in vitro preparations, and all of them showed that $\mathrm{Ca}^{2+}$ activities in the axonal branches of $\alpha^{\prime} / \beta^{\prime}$ neurons in response to a conditioned olfactory stimulus became larger compared with one that was not conditioned. The changes were dependent on proper G-protein signaling in the MB. The importance of these changes in the $\mathrm{Ca}^{2+}$ activity of $\alpha^{\prime} / \beta^{\prime}$ neurons during early memory formation was further tested behaviorally by disrupting G-protein signaling in these neurons or blocking their synaptic outputs during the learning and memory process. Our results suggest that increased $\mathrm{Ca}^{2+}$ activity in response to a conditioned olfactory stimulus may be a neural correlate of early memory in the MB.

Key words: learning and memory; Drosophila; imaging; calcium; olfactory; fluorescence microscopy

\section{Introduction}

Memory is generally considered to be encoded and stored as experience-dependent modifications of synaptic strength and structure (Kandel and Pittenger, 1999; Thompson, 2005). Where in the brain such modifications (i.e., the memory trace) take place is one of the key issues in memory research. In Drosophila, strong evidence suggests that olfactory memory is primarily stored in the mushroom body (MB) (Heisenberg, 2003; Gerber et al., 2004; Davis, 2005), a higher-order brain center that receives olfactory inputs from the antennal lobe $(\mathrm{AL})$, which in turn receives inputs from the olfactory receptor neurons (Hildebrand and Shepherd, 1997; Hallem and Carlson, 2004). For example, chemical ablation of the MB (de Belle and Heisenberg, 1994) abolishes olfactory learning. Furthermore, targeted disruption of the G-protein signaling pathways (Connolly et al., 1996; Ferris et al., 2006) or cAMP cascades (Zars et al., 2000; McGuire et al., 2003) in the MB also disrupts olfactory learning. Finally, temporary blockage of the $\mathrm{MB}$ neuron output disrupts memory acquisition, consolidation, and retrieval (Dubnau et al., 2001; McGuire et al., 2001; Krashes et al., 2007).

\footnotetext{
Received June 29, 2007; revised Feb. 27, 2008; accepted March 17, 2008.

This work was supported by National Institutes of Health Grant 5R01DC005784-05 (Y.Z.) and DART Neuroscience, Inc. We thank Drs. Shouzhen Xia and Tim Tully for $G \alpha_{s}{ }^{*}$ and $G \alpha_{s}{ }^{+}$flies, Dr. Vivian Budnikfor the anti-Trio antibody, and Bito Chen for help using Amira.

*Y.W. and A.M. contributed equally to this work.

Correspondence should be addressed to Dr. Yi Zhong, Cold Spring Harbor Laboratory, P.0. Box 100, Cold Spring Harbor, NY 11724.E-mail: zhongyi@cshl.edu.

Y. Wang's present address: College of Staten Island, City University of New York, Staten Island, NY 10314 D0I:10.1523/JNEUROSCI.2958-07.2008

Copyright $\odot 2008$ Society for Neuroscience $\quad$ 0270-6474/08/264368-09\$15.00/0
}

Following their birth orders, the MB neurons can be divided into three subtypes: the $\gamma, \alpha^{\prime} / \beta^{\prime}$, and $\alpha / \beta$ neurons (Crittenden et al., 1998; Lee et al., 1999). Axons of the $\gamma$ neurons form the horizontal $\gamma$ lobe, whereas those of $\alpha^{\prime} / \beta^{\prime}$ and $\alpha / \beta$ neurons bifurcate and extend into vertical $\alpha^{\prime}$ and $\alpha$ and horizontal $\beta^{\prime}$ and $\beta$ lobes, respectively (see Fig. $1 A$ ). Emerging evidence suggests that different subtypes of $\mathrm{MB}$ neurons may be involved in different phases of the olfactory memory formation. The phases of olfactory memory include short-term memory (STM), middle-term memory (MTM), anesthesia-resistant memory (ARM), and long-term memory (LTM) (Tully et al., 1994). Behavioral studies have suggested that the $\alpha^{\prime}$ and $\alpha$ lobes may be important for LTM (Pascual and Preat, 2001), whereas the $\gamma$ lobe may play an important role in rutabaga-mediated STM (Zars et al., 2000). The outputs from $\alpha^{\prime} / \beta^{\prime}$ neurons are required during acquisition and consolidation, whereas the outputs from $\alpha / \beta$ neurons are required during memory retrieval (Krashes et al., 2007). Consistent with these findings, a functional imaging study has revealed an LTM trace in the $\alpha$ lobe of the $\alpha / \beta$ neurons (Yu et al., 2006). However, although an STM trace has been shown to exist in the AL (Yu et al., 2004), direct demonstration of an STM trace in the $\mathrm{MB}$ is still lacking.

In this study, we visualized learning-induced neural plasticity in the $\mathrm{MB}$ with functional $\mathrm{Ca}^{2+}$ imaging. We found that after a single training, $\mathrm{Ca}^{2+}$ responses in the $\alpha^{\prime} / \beta^{\prime}$ lobes were enhanced for trained odors compared with control odors. This enhancement lasted up to $1 \mathrm{~h}$ and could be blocked by disrupting G-protein signaling in the $\mathrm{MB}$. Restricted disruption of G-protein signaling in the $\alpha^{\prime} / \beta^{\prime}$ neurons or blocking synaptic outputs from these neurons impaired the memory formation. 
These results suggest that the enhanced $\mathrm{Ca}^{2+}$ responses in the $\alpha^{\prime} / \beta^{\prime}$ lobes represent an STM trace.

\section{Materials and Methods}

Transgenic flies and T-maze training. Flies carrying the UAS-G-CaMP transgene were crossed with the MB GAL4 line, OK107. Offspring from this cross (UAS-G-CaMP $\times O K 107)$ have strong G-CaMP expression in the $\mathrm{MB}$ neurons. We also generated homozygous flies carrying both UAS-G-CaMP and an MB Gal4 driver, 238Y. These flies (238Y;UAS-G$C a M P)$ were used interchangeably with $U A S-G-C a M P \times O K 107$ flies. There was no difference in imaging and behavior results obtained from the two lines of flies. To express different variants of $\mathrm{G} \alpha_{\mathrm{s}}$ together with G-CaMP in the MB, UAS-G $\alpha_{s}^{*}$ and $U A S-G \alpha_{s}{ }^{+}$with a $2202 U$ genetic background were crossed with 238Y;UAS-G-CaMP. 2202U× 238Y;UASG-CaMP and UAS- $G \alpha_{s}{ }^{+} \times 238 Y$; UAS-G-CaMP were used as controls for $U A S-G \alpha_{s}{ }^{*} \times 238 Y$; UAS-G-CaMP. $2202 U$ is the isogenized strain of wildtype $w^{1118}$.

Flies were trained in a standard T-maze as described previously (Tully and Quinn, 1985). Briefly, $\sim 120$ flies were sequentially exposed to a positive conditioned stimulus $\left(\mathrm{CS}^{+}\right)$odor paired with footshock and then a negative conditioned stimulus $\left(\mathrm{CS}^{-}\right)$odor without footshock. Flies were either tested immediately after training ( 3 min memory) or, with one fly caught alive for imaging, tested at $2 \mathrm{~h}$ after training. After the training, the flies were kept on standard Drosophila medium at room temperature. During testing, flies were exposed simultaneously to the $\mathrm{CS}^{+}$and $\mathrm{CS}^{-}$odors in a T-maze. A performance index (PI) was calculated from the distribution of flies in the two T-maze arms. A 50:50 distribution (no memory) gives rise to a PI score of 0 , and a 0:100 distribution away from the $\mathrm{CS}^{+}$gives rise to a PI of 100. A 1000-fold dilution of octanol (OCT) and 2500-fold dilution of benzaldehyde (BA) in mineral oil were used. For control cases, flies were sequentially exposed to a $\mathrm{CS}^{+}$odor, electric shock, and a $\mathrm{CS}^{-}$odor, each for $1 \mathrm{~min}$, with a $1 \mathrm{~min}$ interval in between.

Living fly imaging. The captured fly was prepared for live imaging as described previously with some modifications (Wang et al., 2001). Briefly, the fly was immobilized in a micropipette tip with its head exposed. A plastic coverslip with a small window was placed over the head and sealed onto the fly around the edges of the window with dental wax. After covering the head with adult fly saline [in mM: $115 \mathrm{NaCl}, 5 \mathrm{KCl}, 1.5$ $\mathrm{CaCl}_{2} \cdot 2 \mathrm{H}_{2} \mathrm{O}, 4 \mathrm{MgCl}_{2} \cdot 6 \mathrm{H}_{2} \mathrm{O}, 4 \mathrm{NaHCO}_{3}, 1 \mathrm{NaH}_{2} \mathrm{PO}_{4} \cdot 1 \mathrm{H}_{2} \mathrm{O}, 5$ trehalose, 75 sucrose, and $5 \mathrm{~N}$-Tris (hydroxymethyl) methyl-2aminoethanesulfonic acid, $\mathrm{pH} 7.1,356$ mOsm], a tiny hole was cut through the cuticle to make the MB accessible for imaging.

To train a fly that was immobilized for imaging, the preparation described above was modified slightly. The micropipette tip that held the fly had a metal electrode inserted through which the electric shock could be delivered to the fly's abdomen. To ensure the shocking of the abdomen, the surface of the metal electrode was covered with electro-conductive gel. Training was done to the single fly following a procedure similar to the one used in the T-maze, except that $120 \mathrm{~V}$ square pulses were used instead of standard $60 \mathrm{~V}$ pulses. For unpaired control experiments, flies were sequentially exposed to BA, electric shock, and OCT, each for $1 \mathrm{~min}$, with a $1 \mathrm{~min}$ interval in between. Odors were diluted in mineral oil at the same concentrations used in the T-maze training and were delivered to the fly's free-moving antennae by an air stream $(400 \mathrm{ml} / \mathrm{min})$ bubbled through mineral oil. Electric shock evoked very small responses in the $\mathrm{MB}$, which could only be revealed after a spectral analysis of the averaged response time course.

A custom-built two-photon laser-scanning microscope was used. The objective was a $60 \times$ water-immersion lens $(0.9$ numerical aperture; Olympus, Center Valley, PA). The laser source was a Chameleon Ti: sapphire laser (Coherent, Santa Clara, CA) tuned to wavelength $(\lambda) 910$ $\mathrm{nm}$. Images were acquired at $\sim 5 \mathrm{~Hz}(0.2 \mathrm{~s}$ per image). Recordings were taken from single optical sections of the MB. At the end of each experiment, an XYZ scan ( $z$ step of $1 \mu \mathrm{m}$ ) was always performed to ascertain the identification of the MB substructures. The three-dimensional MB structure was reconstructed from such scans using Amira (TGS, San Diego, CA).

Isolated brain preparation. The brain was isolated from the cuticle with the antennal nerves (ANs) and the ventral nerve cord (VNC) attached and was pinned down on a thin Sylgard slab in a Petri dish. The electric stimulation to the AN and VNC were accomplished by two suction electrodes, both with a train of 10 pulses (single pulse duration, $1 \mathrm{~ms}$ ) at 100 $\mathrm{Hz}$ using a Grass S88 stimulator (Grass Technologies, West Warwick, RI). For paired stimulation, 12 trains at an interval of $5 \mathrm{~s}$ were delivered simultaneously to the AN and VNC. For backward pairing, the stimulation of the VNC preceded that of the AN by $30 \mathrm{~s}$. For unpaired stimulation, there was a 1 min interval between AN stimulation and VNC stimulation. Responses in the MB evoked by the stimulation of the AN was much bigger than those evoked by the in vivo odor stimulations. To ensure the response to $\mathrm{AN}$ and VNC stimulation was not saturated, voltages of stimuli were chosen to give a response that was below $30 \%$ of the maximum responses.

Image analysis. The $\Delta F$ images were generated as described previously (Wang et al., 2004). Briefly, frames acquired before the onset of odor stimulation were averaged, and the SEM of the background fluorescence fluctuation at each pixel was calculated. The averaged background image was subtracted from the average of a few frames at the peak of the odor response to obtain the $\Delta F$ image. A pixel-by-pixel threshold of two times the SEM of the background fluorescence fluctuation was applied to the $\Delta F$ image. Regions of interest were identified based on the basal fluorescence of G-CaMP showing characteristic shape and location of each region of interest. Each lobe was clearly identifiable at the tip region, and the $x-y-z$ stack images were used to follow each lobe to the depths of recordings. The ratio of the peak response amplitude to the $\mathrm{CS}^{+}$odors versus $\mathrm{CS}^{-}$odors were log transformed (base 10) to normalize the distribution of the ratio, and statistical tests were performed on log transformed values. Unless noted otherwise, data were analyzed by ANOVA followed by Tukey-Kramer honestly significant difference post hoc tests, and the critical values for individual comparisons were adjusted to maintain an experiment-wise error rate of $\alpha=0.05$.

Immunohistochemistry. Brains were dissected in PBS and fixed in 4\% paraformaldehyde (in PBS) at room temperature for $10 \mathrm{~min}$. After vacuuming twice in PBST (PBS with $0.2 \%$ Triton X-100) and $4 \%$ paraformaldehyde for $10 \mathrm{~min}$, the brains were blocked with $10 \%$ normal goat serum (NGS) on ice for $2 \mathrm{~h}$. The brains were then incubated overnight at $4^{\circ} \mathrm{C}$ with mouse 1D4 anti-Fasciclin II antibody (supernatant, 1:20 diluted in PBS with $0.25 \%$ Triton X-100, 1\% NGS, and $0.02 \% \mathrm{NaN}_{3}$; DSHB, University of Iowa, Iowa City, IA). After washing four times in PBS, the brains were incubated overnight at $4^{\circ} \mathrm{C}$ with goat anti-mouse IgG conjugated with Alexa Fluor 647 (1:200 diluted in PBS with 0.25\% Triton $\mathrm{X}-100,1 \% \mathrm{NGS}$, and $0.02 \% \mathrm{NaN}_{3}$; Invitrogen, Eugene, OR). The brains were then washed and mounted in FocusClear (Pacgen, Vancouver, British Columbia, Canada). Confocal images of the brain were taken with an LSM 510 confocal microscope (Carl Zeiss MicroImaging, Thornwood, NY).

\section{Results}

We used a genetically engineered calcium sensor, G-CaMP, to monitor neuronal activities (Wang et al., 2003, 2004). Expression of the G-CaMP transgene was targeted to the MB using the GAL4-UAS system (Brand and Perrimon, 1993). Two GAL4 lines showing strong expression in the MB, OK107 and 238Y (Connolly et al., 1996), were used interchangeably in this study. A fly was restrained in a holder fashioned from a micropipette tip, and a window was opened in the head for imaging with a two-photon laser-scanning microscope. The basal fluorescence of the G-CaMP allowed identification of the MB structure (Fig. S1, available at www.jneurosci.org as supplemental material). Recordings were made from optical sections in regions of the calyx (the dendritic region of MB neurons) and the vertical $\alpha^{\prime} / \alpha$ and horizontal $\beta^{\prime} / \beta / \gamma$ lobes as illustrated in Figure $1 A$. Delivery of an odor evoked increases in G-CaMP fluorescence in the calyx and axonal branches (Fig. $1 B, C$ ). The majority of this rise in G-CaMP fluorescence is likely to be caused by an influx of extracellular $\mathrm{Ca}^{2+}$, because depletion of the intracellular $\mathrm{Ca}^{2+}$ stores did not diminish G-CaMP signals evoked by stimulation of the olfactory pathway (Fig. S2, available at www.jneurosci.org as supplemental material). In the axonal branches, odor-evoked calcium re- 
sponses were much higher in the $\alpha^{\prime}$ and $\beta^{\prime}$ lobes than in $\alpha, \beta$, and $\gamma$ lobes (Figs. 1, 2).

We trained flies with an olfactory associative learning paradigm that has been most extensively characterized in flies (Tully and Quinn, 1985). In this paradigm, flies learn to avoid an odor that is associated with electric shock. We exposed flies first to an odor $\left(\mathrm{CS}^{+}\right)$, BA, which was paired with electric shock to the flies, followed by exposure to another odor $\left(\mathrm{CS}^{-}\right)$, OCT, without electric shock. In control groups, flies were exposed to the two odors and electric shock sequentially without pairing (Fig. 2A). After training, a fly was captured randomly without anesthetization and prepared for live imaging of $\mathrm{Ca}^{2+}$ responses in the $\mathrm{MB}$ to the $\mathrm{CS}^{+}$and $\mathrm{CS}^{-}$odors. The cohort flies were tested behaviorally after the completion of live imaging to demonstrate the memory retained that was evidenced by their ability to avoid the $\mathrm{CS}^{+}$odor (Fig. 2A). At $2 \mathrm{~h}$ after training, the flies still retained a significant level of memory. Constrained by the time needed to prepare a fly for live imaging, G-CaMP responses to both the $\mathrm{CS}^{+}$and $\mathrm{CS}^{-}$ odors were recorded in captured flies at $\sim 60$ min after training. In trained flies, responses to BA relative to those to OCT were much enhanced in $\alpha^{\prime} / \beta^{\prime}$ lobes compared with control flies (Fig. $2 C-E$, Control and BA-trained). The time course of the G-CaMP response seemed not to have changed by training (Fig. $2 B-D$ ). No relative changes in responses to BA and OCT in the calyx and the $\alpha / \beta$ and $\gamma$ lobes were observed (Fig. $2 B-E$ ). These findings were confirmed by switching the two odors in the T-maze training with $\mathrm{OCT}$ as $\mathrm{CS}^{+}$and BA as $\mathrm{CS}^{-}$. After such training, activities in $\alpha^{\prime} / \beta^{\prime}$ lobes in response to OCT were enhanced compared with BA (Fig. 2C,D,F). No activity changes were observed in the calyx and the $\gamma$ and $\alpha / \beta$ lobes (Fig. $2 B-D, F$ ). Our recordings covered large fractions of the calyx, $\beta^{\prime}, \beta$, and $\gamma$ lobes, although in the vertical lobes $\left(\alpha^{\prime}\right.$ and $\alpha$ ), recordings were restricted around the tip region of the $\alpha^{\prime}$ lobe (Table S1, available at www.jneurosci.org as supplemental material), where most of the G-CaMP responses to olfactory pathway stimulation were found (data not shown). It is therefore unlikely that the lack of changes in G-CaMP response in the calyx and the $\gamma$ and $\alpha / \beta$ lobes is attributable to inadequate sampling. We also used the Gal4 line G0050 to express G-CaMP specifically in $\alpha^{\prime} / \beta^{\prime}$ neurons (Lin et al., 2007) and performed the same experiment to confirm the learning-induced increase in the odor-evoked activity in these lobes (Fig. S3, available at www.jneurosci.org as supplemental material). Results from these experiments confirmed that activities in $\alpha^{\prime} / \beta^{\prime}$ lobes in response to a $\mathrm{CS}^{+}$odor was enhanced compared with a $\mathrm{CS}^{-}$ odor after the training (Fig. S3, available at www.jneurosci.org as supplemental material).

However, in the first preparation, activity in the MB immediately after learning could not be examined, and odor responses before and after training in a single fly could not be compared. To overcome these problems, we adopted a preparation that allowed training while a fly was being imaged (Yu et al., 2004). The fly was immobilized, and the head capsule opened up for $\mathrm{Ca}^{2+}$ imaging. Electric shock was delivered to the fly with a metal wire inserted under its abdomen. The fly was first exposed to BA paired with electric shock, followed by exposure to OCT without the shock. We examined responses to both odors in the vertical lobes before and after training. Odor-evoked responses were mostly in the $\alpha^{\prime}$ lobe as were shown in the first preparation (Fig. 3). We found a trend of BA response to become larger and OCT response to become smaller after the training, but this trend was not statistically significant $(p>0.05)$. When we compared the ratio of $\mathrm{BA}$ response to OCT response, we found a statistically significant
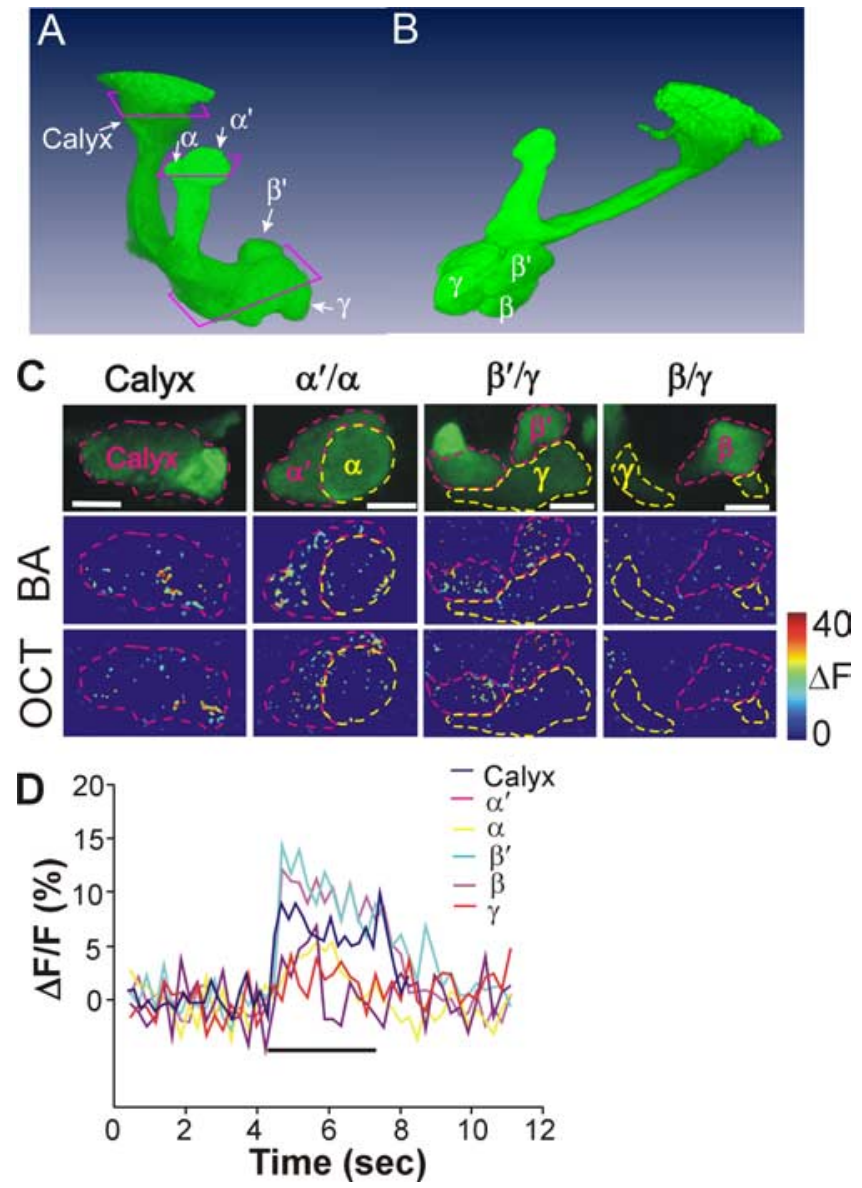

Figure 1. Odor-evoked G-CaMP responses in the MB. $A$, MB structure reconstructed from two-photon scanning stacks of a living fly brain expressing G-CaMP driven by $0 \mathrm{~K} 107$ (frontal view). The MB was identified by the basal G-CaMP fluorescence. Only the right half of the MB is shown. The arrowheads identify the axonal branches of subtypes of MB neurons. The rectangular outlines indicate places where recordings were taken to monitor activities in the calyx and different axonal branches. $\boldsymbol{B}$, Sagittal view of the reconstructed MB structure. The $\beta$ lobe lies beneath the $\gamma$ and $\beta^{\prime}$ lobes. C, G-CaMP responses evoked by BA (second row) and OCT (third row) in the calyx and axonal branches of the MB. $\Delta$ Fimages of the G-CaMP response are shown. The top row shows basal G-CaMP fluorescence that identifies the structures from which recordings were made. $\boldsymbol{D}$, Time course of the G-CaMP response to BA in the calyx and axonal branches of the MB outlined in C. The time course of G-CaMP response in only one of the sections of the $\gamma$ lobe shown in $\boldsymbol{C}$ is plotted. The bar shows the duration of odor stimulation. Scale bar: vertical lobes, $10 \mu \mathrm{m}$; calyx and horizontal lobes, $20 \mu \mathrm{m}$.

increase in the $\alpha^{\prime}$ lobe 60 min after training (Fig. 3B). There was also a slight increase at $30 \mathrm{~min}$, although not statistically significant. No change in the response to BA and OCT was found in the control flies that were exposed sequentially to BA, electric shock, and OCT (repeated-measures ANOVA; $F_{(2,8)}=0.38 ; p=$ $0.6933)$. For both trained and control flies, no changes were observed in the $\alpha$ lobe. This is consistent with the finding obtained from flies trained in the T-maze.

In the second preparation, an immediate change in $\mathrm{Ca}^{2+} \mathrm{ac}-$ tivities could have been masked by considerable brain movement that reduces the signal-to-noise ratio. To minimize the muscle movement, we developed another preparation that allowed us to investigate associative conditioning. We stripped bare the living fly preparation with only the brain left and the AN and VNC attached. We paired electric stimulations of the AN, which conducts olfactory input from the periphery, with that of the VNC, which may convey sensation of electric shock from the body to the brain, and imaged neuronal activities in the $\mathrm{MB}$ in response to 

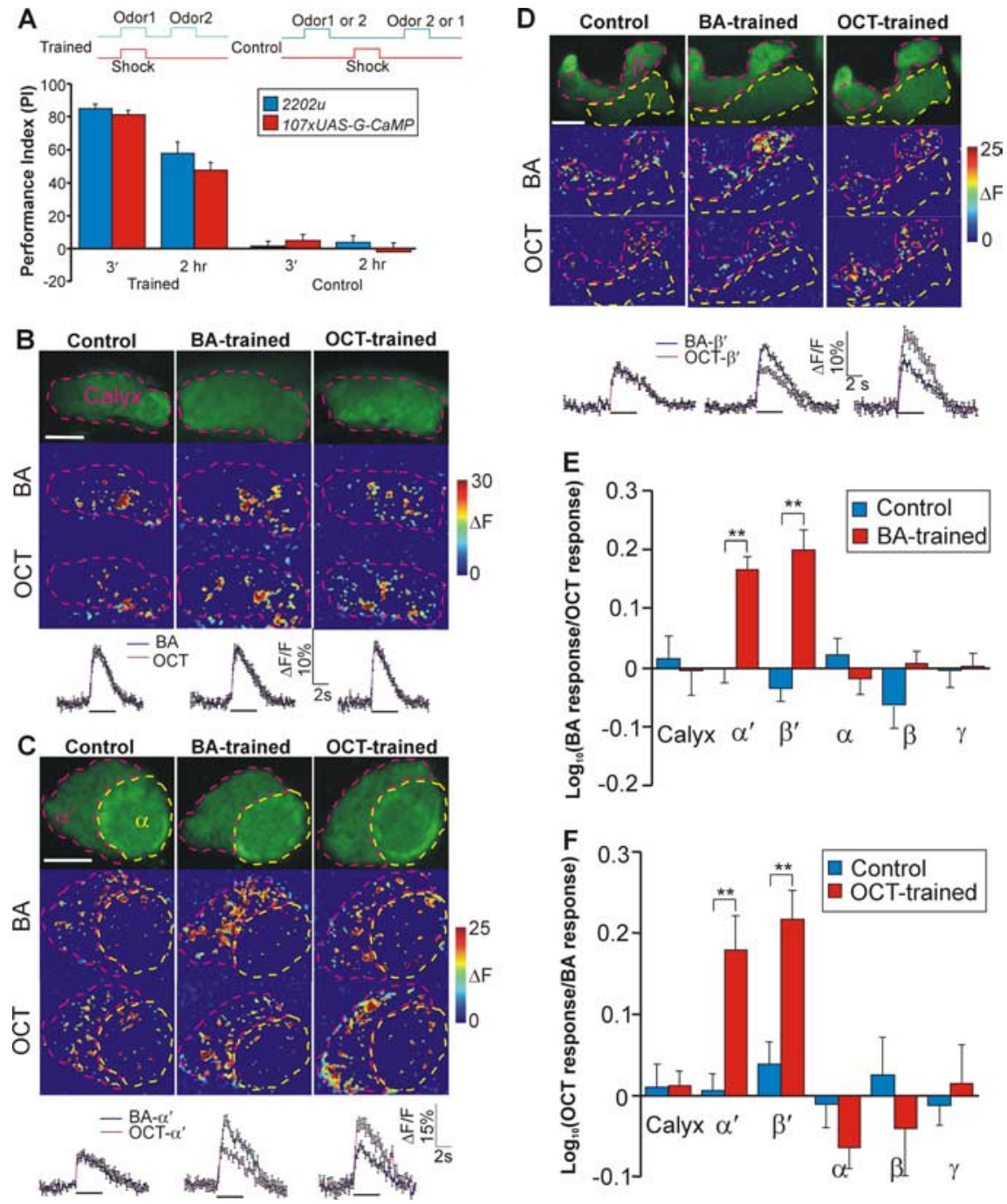

Figure 2. Learning-induced enhancement in G-CaMP response to $\mathrm{CS}^{+}$odors in the MB $\alpha^{\prime}$ and $\beta^{\prime}$ lobes after olfactory training. $\boldsymbol{A}$, Memory performance of flies immediately after training ( $3 \mathrm{~min}$ ), or $2 \mathrm{~h}$ after. Flies were forced to choose between the $\mathrm{CS}^{+}$and $\mathrm{CS}^{-}$. A score of 100 indicates all flies avoiding the $\mathrm{CS}{ }^{+}$. The training protocols are illustrated on top. Flies were either trained with $\mathrm{BA}\left(\mathrm{BA} \mathrm{as} \mathrm{CS}^{+}\right.$and $0 \mathrm{CT}$ as $C S^{-}$) or $0 \mathrm{CT}\left(0 \mathrm{CT}\right.$ as $\mathrm{CS}^{+}$and $\mathrm{BA}$ as $\left.C S^{-}\right)$. In training, $\sim 120$ flies were first exposed to the $\mathrm{CS}^{+}$odor paired with electric shock for $1 \mathrm{~min}$. After resting for $1 \mathrm{~min}$, the flies were exposed to the $\mathrm{CS}^{-}$odor for 1 min. In control, about the same number of flies was sequentially exposed to $\mathrm{CS}^{+}{ }^{+}$, electric shock, and CS ${ }^{-}$, each for 1 min, with a 1 min interval. After the training, the flies were either tested immediately for 3 min memory, or with a fly caught for live imaging, the rest were tested $2 \mathrm{~h}$ after the training. $\boldsymbol{B}-\boldsymbol{D}$, The G-CaMP response to BA (second row) and OCT (third row) in the calyx $(\boldsymbol{B}), \alpha^{\prime} / \alpha(\boldsymbol{C})$, and $\beta^{\prime} / \gamma(D)$ lobes of the control (first column), BA-trained (second column), and OCT-trained (third column) flies. Recordings were made $\sim 60$ min after the training. In the lobes, responses to BA and OCT were mostly concentrated in the $\alpha^{\prime} / \beta^{\prime}$ lobes $(\boldsymbol{C}, \boldsymbol{D})$. The responses to the $\mathrm{CS}^{+}$odors were specifically enhanced relative to the responses to the CS ${ }^{-}$odors in the $\alpha^{\prime}$ and $\beta^{\prime}$ lobes after training $(\boldsymbol{C}, \boldsymbol{D})$. No change was observed in the response to the $\mathrm{CS}^{+}$odors relative to $\mathrm{CS}^{-}$odors in flies trained with either $\mathrm{BA}$ or OCT in the calyx $(\boldsymbol{B}), \alpha$ lobe $(\boldsymbol{C})$, and $\gamma$ lobe $(\boldsymbol{D})$. The top rows show G-CaMP basal fluorescence that identified structures from where recordings were made. The bottom rows show averaged time courses of the $\mathrm{G}$-CaMP responses $(\triangle F / F)$ to $B A$ and $O C T$ from the corresponding group of flies in the MB regions outlined by the dashed lines. The number of flies used in each averaged time course ranged from 7 to 10 . The bars indicate the duration of odor stimulation. $\boldsymbol{E}, \boldsymbol{F}$, Enhanced responses to the $\mathrm{CS}^{+}$relative to the $\mathrm{CS}^{-}$odors in the $\alpha^{\prime} / \beta^{\prime}$ lobes in BA-trained $(\boldsymbol{E})$ and OCT-trained $(\boldsymbol{F})$ flies. Recordings were made in the calyx, $\alpha^{\prime}, \alpha, \beta^{\prime}, \beta$, and $\gamma$ lobes. The $\mathrm{Log}$ ratios of the $C S^{+}$response to the $\mathrm{CS}^{-}$response were calculated using the peak amplitudes of the responses. In $\boldsymbol{E}, n=7$ and 9 for control and BA-trained flies, respectively. In $\boldsymbol{F}, n=8$ and 10 for control and 0CT-trained flies, respectively. Asterisks indicate significance between trained and control flies $\left({ }^{*} p<0.05\right.$; ${ }^{* *} p<0.01$ ). Error bars represent SEM. Scale bars: vertical lobes, $10 \mu \mathrm{m}$; calyx and horizontal lobes, $20 \mu \mathrm{m}$.

AN stimulation before and after pairing. Although the pathway that carries the electric shock information to the brain is not clear, we think that the stimulation of the VNC provides a good approximation. It evoked responses in the MB similar to those shown by others when the electric shock was delivered to the abdomen (Yu et al., 2006). Stimulations of the AN or VNC both evoked activities in the MB calyx and lobes (Fig. 4A). Brain movement was drastically reduced in this preparation as shown by the smoother baseline in the time course plot (Fig. 4A). A paired stimulation of the AN and VNC that mimicked the time course of the olfactory training in the T-maze enhanced $\mathrm{Ca}^{2+}$ responses in $\alpha^{\prime} / \beta^{\prime}$ lobes to a subsequent $\mathrm{AN}$ stimulation at $\sim 3$ min after pairing (Fig. $4 B, C$ ). No such enhancements were detected in the calyx and the $\gamma$ and $\alpha / \beta$ lobes (Fig. 4B). Backward pairing in which VNC stimulation preceded AN stimulation, or sequential stimulation of the AN and the VNC without any overlap, did not change responses to AN stimulation (Fig. $4 D, E$ ). This shows that the enhanced responses to $\mathrm{AN}$ stimulation is specific to the paired stimulation of the AN and VNC.

It has been shown that associative olfactory learning can be disrupted by expressing in the $\mathrm{MB}$ a mutant form of the stimulatory heterotrimeric G-protein $\alpha$ subunit $\left(\mathrm{G} \alpha_{\mathrm{s}}{ }^{*}\right)$ that constitutively activates the adenylyl cyclase and impairs the cAMP pathway (Connolly et al., 1996). The same study also showed that expression of the wild-type $\mathrm{G} \alpha_{\mathrm{s}}\left(\mathrm{G} \alpha_{\mathrm{s}}{ }^{+}\right)$does not affect learning. If a pairing of the $\mathrm{AN}$ and VNC stimulation truly mimicked olfactory learning, the pairing-induced enhancement of responses to AN stimulation in $\alpha^{\prime} / \beta^{\prime}$ lobes should be blocked by the expression of $\mathrm{G} \alpha_{\mathrm{s}}{ }^{*}$ in the $\mathrm{MB}$, but not with the expression of $\mathrm{G}_{\mathrm{s}}{ }^{+}$in the MB. We expressed both G-CaMP and $\mathrm{G} \alpha_{\mathrm{s}}{ }^{*}$ or $\mathrm{G} \alpha_{\mathrm{s}}{ }^{+}$ in the $\mathrm{MB}$ and compared responses to $\mathrm{AN}$ stimulation before and after the pairing in the MB. Although responses to AN stimulation was enhanced after the pairing in $\alpha^{\prime} / \beta^{\prime}$ lobes in control flies and in flies expressing $\mathrm{G} \alpha_{\mathrm{s}}{ }^{+}$, such enhancement was absent in flies expressing $\mathrm{G} \alpha_{\mathrm{s}}{ }^{*}$ (Fig. 5). So, indeed, the disruption of the G-protein signaling pathway in the $\mathrm{MB}$ abolished the pairing-induced enhancement of responses to AN stimulation in $\alpha^{\prime} / \beta^{\prime}$ lobes, further supporting the idea that the pairing-induced enhancement is a result of an associative process.

The enhanced G-CaMP response to trained odors compared with control odors in $\alpha^{\prime} / \beta^{\prime}$ lobes after training indicates that $\alpha^{\prime} / \beta^{\prime}$ neurons may be involved in the learning and memory process. This is consistent with a recent finding that the output from $\alpha^{\prime} / \beta^{\prime}$ neurons are required for acquisition and consolidation of $3 \mathrm{~h}$ memory (Krashes et al., 2007). To further demonstrate the participation of $\alpha^{\prime} / \beta^{\prime}$ neurons in early forms of memory, we disrupted the G-protein signaling only in $\alpha^{\prime} / \beta^{\prime}$ neurons by targeting the constitutively active $\mathrm{G} \alpha_{\mathrm{s}}{ }^{2}$ to these neurons with the GAL4 line G0050 (Lin et al., 2007) (Fig. 


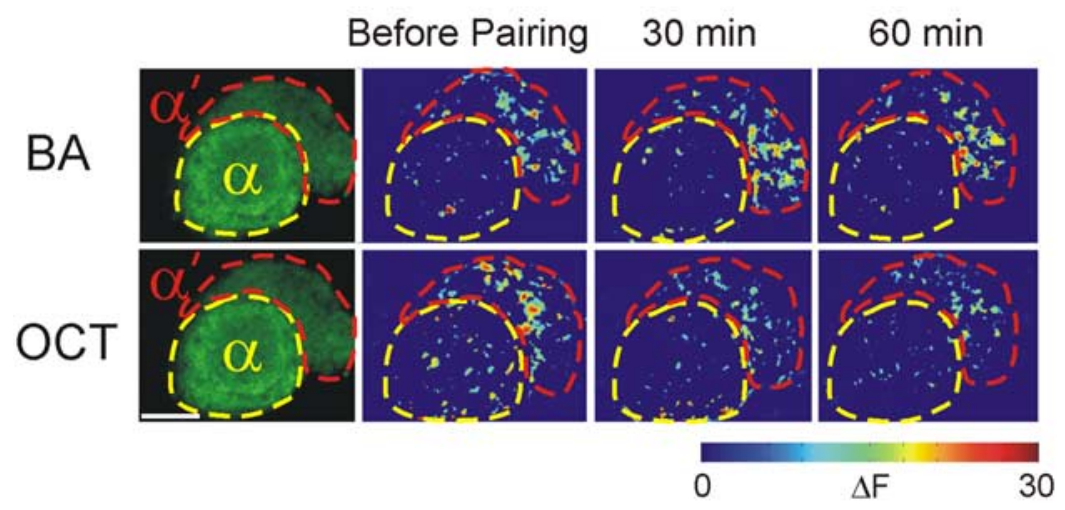

\section{B}



Figure 3. Pairing BA with electric shock changes the ratio of G-CaMP response to BA and OCT in the $\alpha^{\prime}$ lobe of the MB. $A$, First column, The basal fluorescence of G-CaMP expressed with OK107-GAL4 in the $\alpha$ (surrounded by yellow dotted lines) and $\alpha^{\prime}$ (surrounded by red dotted lines) lobes of the MB (scale bar, $10 \mu \mathrm{m}$ ). Note that the lateral is to the right in these images. Second to fourth columns, Changes in G-CaMP fluorescence $(\Delta F)$ in response to 3 s presentations of BA (top row) and OCT (bottom row) at different time points before and after the pairing of BA with electrical shock (second column, before pairing; third column, 30 min after the pairing; fourth column, $60 \mathrm{~min}$ after the pairing). Most of the changes in G-CaMP fluorescence were seen in the $\alpha^{\prime}$ lobe. In this example, after the pairing, responses to $B A$ became slightly bigger, whereas responses to $0 C T$ became slightly smaller. $B, \log _{10}$ of the response to $B A$ relative to the response to $0 C T$ changed significantly after the pairing (one-way ANOVA; $F_{(2,15)}=3.88 ; p=0.0439$ ). $\log _{10}$ of the response to $B A$ relative to the response to 0 CT was calculated in each fly at various time points before and after the pairing of $B A$ with electrical shock, and a mean value for six flies is plotted for each time point. Comparison of individual means showed that the $\log _{10}$ of the response ratio was significantly greater at $60 \mathrm{~min}$ after the pairing compared with the presentation before the pairing (Tukey-Kramer adjusted, ${ }^{*} p=0.0328$ ). The peak amplitudes of the responses were used for the calculation of the response ratio. Error bars represent SEM.

$6 A)$. Memory was tested immediately after a single-cycle training using the standard T-maze training paradigm. As shown in Figure $6 B$, disruption of the G-protein signaling in $\alpha^{\prime} / \beta^{\prime}$ neurons partially impaired memory immediately after the training. We also blocked outputs from the $\alpha^{\prime} / \beta^{\prime}$ neurons with a temperaturesensitive dynamin, $\mathrm{Shi}^{\text {ts }}$, which can disrupt synaptic transmission in a dominant-negative way at restrictive temperatures (Kitamoto, 2001). Similar to disrupting the G-protein signaling, blockade of output from $\alpha^{\prime} / \beta^{\prime}$ neurons with $\mathrm{Shi}{ }^{\text {ts }}$ by raising the temperature to $32^{\circ} \mathrm{C}$ partially impaired immediate memory (Fig. $6 C)$. We further examined the involvement of $\alpha^{\prime} / \beta^{\prime}$ neurons in $1 \mathrm{~h}$ memory which, like $3 \mathrm{~h}$ memory, comprises MTM and ARM. Consistent with the finding that the output from $\alpha^{\prime} / \beta^{\prime}$ neurons is required for consolidation of $3 \mathrm{~h}$ memory, blocking the output from $\alpha^{\prime} / \beta^{\prime}$ neurons after training impaired $1 \mathrm{~h}$ memory (Fig. $6 D)$. Task-relevant sensory-motor responses were not altered by either the expression of $\mathrm{G} \alpha_{\mathrm{s}}{ }^{*}$ in the $\alpha^{\prime} / \beta^{\prime}$ neurons or disrupting the output from $\alpha^{\prime} / \beta^{\prime}$ neurons with Shi ${ }^{\text {ts }}$ at the restrictive temperature (Table S2, available at www.jneurosci.org as supplemental material).

\section{Discussion}

We used functional $\mathrm{Ca}^{2+}$ imaging in three different preparations to search for memory traces in the MB after a single-cycle training that produces STM, MTM, and ARM. Flies were trained in groups in the standard T-maze or individually while held immobilized. These two different settings in training both led to enhanced $\mathrm{Ca}^{2+}$ activity in response to trained odors in the axonal branches of the $\alpha^{\prime} / \beta^{\prime}$ neurons. The enhancement was specific to the odor that was paired with electric shock and was detected at $\sim 1$ hour after conditioning. The semi-in vivo preparation that mimicked the single-cycle olfactory training with paired stimulation of the AN and VNC led to a similar observation. In addition, an immediate enhancement in $\mathrm{Ca}^{2+}$ response in $\alpha^{\prime} / \beta^{\prime}$ lobes after the paired event was observed in this preparation. Targeted disruption of the G-protein signaling in the MB blocked the enhancement of $\mathrm{Ca}^{2+}$ response after paired stimulation of $\mathrm{AN}$ and VNC. These results indicate that the enhanced $\mathrm{Ca}^{2+}$ response in $\alpha^{\prime} / \beta^{\prime}$ lobes after conditioning may represent a memory trace associated with early forms of memory. This was further confirmed in a behavioral experiment in which the $3 \mathrm{~min}$ and $1 \mathrm{~h}$ memory was impaired by disrupting the G-protein signaling in the $\alpha^{\prime} / \beta^{\prime}$ neurons or blocking synaptic output from these neurons.

Our finding does not preclude the possible existence of memory traces in other output branches of the MB neurons. There are a number of possibilities why the enhanced $\mathrm{Ca}^{2+}$ response to trained odors was only observed in axonal branches of the $\alpha^{\prime} / \beta^{\prime}$ neurons. Memory traces in the $\alpha / \beta$ and $\gamma$ neurons may appear in forms other than alteration of $\mathrm{Ca}^{2+}$ response. It is known that a $\mathrm{Ca}^{2+}$-independent PKC signaling can potentiate synaptic transmission presynaptically (Stevens and Sullivan, 1998). Another possibility is that for $\alpha / \beta$ and $\gamma$ neurons, memory traces may occur postsynaptically in downstream neurons. Postsynaptic mechanisms play a key role in long-term potentiation and longterm depression (Malinow and Malenka, 2002). A third possibility is that the low G-CaMP responses in $\alpha / \beta$ and $\gamma$ lobes are making it difficult to detect changes in these lobes (Figs. 1, 2). Activities in MB neurons are sparse (Perez-Orive et al., 2002; Wang et al., 2004), and the $\gamma$ neurons tend to show a lower probability of response (Turner et al., 2008). Changes in a small amount of activity may be hard to detect with our imaging approach (Reiff et al., 2005). Finally, learning may lead to changes in the fine temporal pattern of neuronal activities in the $\mathrm{MB}$ that cannot be detected by $\mathrm{Ca}^{2+}$ imaging.

Olfactory learning and memory in Drosophila seem to involve multiple brain structures in parallel and sequential pathways. Blocking the output from the MB impairs retrieval of memory at multiple stages that encompass STM, MTM, ARM, and LTM 
A
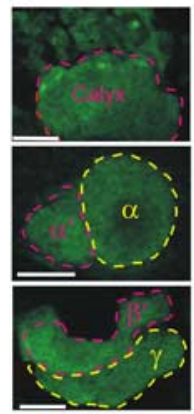

VNC

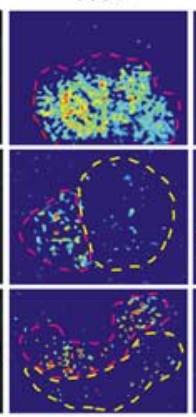

AN



After



D

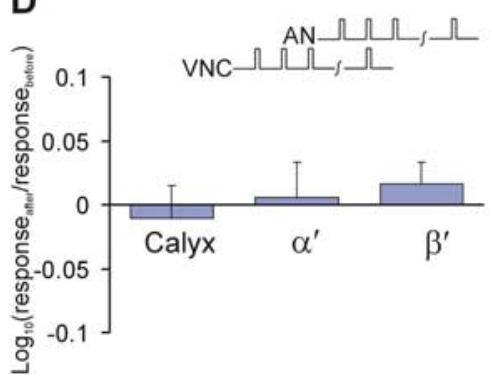

$\mathrm{VNC}+\mathrm{AN}$

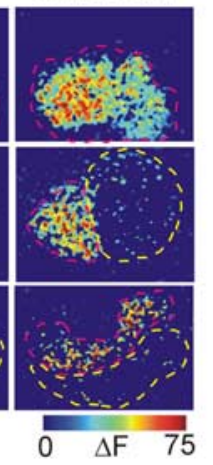

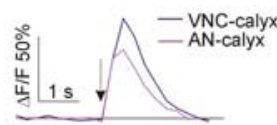
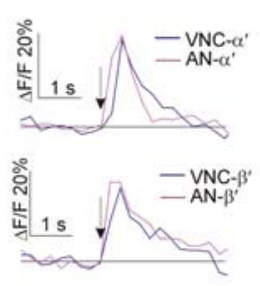

C





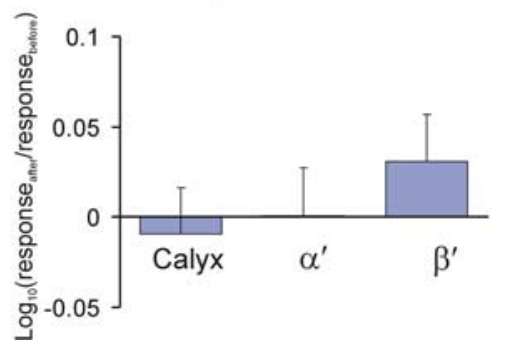

Figure 4. Enhancement in response to AN stimulation in the $\alpha^{\prime} / \beta^{\prime}$ lobes after paired electric stimulation of the AN and VNC. $A$, Responses to stimulation of the AN and VNC in the MB. The basal G-CaMP fluorescence images (first column) reveals sections of the calyx, the vertical lobes ( $\alpha^{\prime}$ and $\alpha$ ), and the horizontal lobes $\left(\beta^{\prime}\right.$ and $\gamma$ ) where recordings were made. Second column, Response to VNC stimulation; third column, response to AN stimulation; fourth column, response to stimulation of both the AN and VNC; fifth column, time course of responses to AN and VNC stimulation in MB regions outlined by dashed lines. Arrows indicate when electric stimulations were delivered. Stimulations of the AN and VNC both evoked responses in the MB calyx and the vertical and horizontal lobes. Similar to responses to odor stimulation in the in vivo fly preparations, responses to AN and VNC stimulation in the isolated brain were mostly concentrated in the $\alpha^{\prime} / \beta^{\prime}$ lobes. $\boldsymbol{B}$, Responses to stimulation of the AN before and 5 min after paired electrical stimulation of the AN and VNC. Responses to AN stimulation before and 5 min after paired stimulation of the AN and VNC from the sections in the left column are displayed as $\Delta$ Fimages (two right columns). For stimulation of the AN and VNC, a train of 10 pulses (single-pulse duration, $1 \mathrm{~ms}$ ) at $100 \mathrm{~Hz}$ was used. For paired stimulation, 12 trains at an interval of $5 \mathrm{~s}$ were delivered simultaneously to the AN and VNC. C, Summary of the changes in responses to AN stimulation (expressed as $\log _{10}$ of response after pairing relative to that before) at different time points after paired stimulation of the AN and VNC. Significant enhancement in the response to AN stimulation occurred in the $\alpha^{\prime} / \beta^{\prime}$ lobes ( $t$ test, $\left.{ }^{*} p<0.001 ; n=10\right)$. The peak amplitudes of responses were used for the calculation of the response ratio. $\boldsymbol{D}, \boldsymbol{E}$, Summary of the changes in responses to AN stimulation after a backward pairing of stimuli $(\boldsymbol{D})$ to the AN and VNC (stimulation of the VNC preceded that of the AN by $30 \mathrm{~s}$ ) or sequential stimulation of the AN and VNC (1 min interval) without overlap (E). No enhancement in responses to AN stimulation was observed $(n=9)$. Error bars represent SEM. Scale bars: (in $\boldsymbol{A}, \boldsymbol{B})$ vertical lobes, $10 \mu \mathrm{m}$; calyx and horizontal lobes, $20 \mu \mathrm{m}$.

(Dubnau et al., 2001; McGuire et al., 2001; Isabel et al., 2004). This requirement of the $\mathrm{MB}$ output has been further dissected and assigned to different subtypes of $\mathrm{MB}$ neurons for different stages of memory processing. The initial study with the Shi ${ }^{\text {ts }}$ has indicated that retrieval of $3 \mathrm{~min}$ and $3 \mathrm{~h}$ memory may be exclusively through $\alpha / \beta$ neurons (McGuire et al., 2001). The most recent study on $3 \mathrm{~h}$ memory confirmed the role for $\alpha / \beta$ neurons in retrieval and further revealed that output of the $\alpha^{\prime} / \beta^{\prime}$ neurons is required for acquisition and consolidation (Krashes et al., 2007). Several places outside the MB are also involved in olfactory learning and memory. Output from a pair of neurons called dorsal paired medial (DPM) neurons that innervate the entire $\mathrm{MB}$ lobes are required for $3 \mathrm{~h}$ memory in a time window of 30-150 min after conditioning (Keene et al., 2004, 2006; Yu et al., 2005). One of the NMDA receptor subunits, NR1, which is preferentially expressed in a small number of neurons that innervate the $\mathrm{MB}$ dendritic region, affects learning and LTM consolidation (Xia et al., 2005). Several genes expressed in a small number of neurons outside the $\mathrm{MB}$ were identified in a large-scale mutagenesis to affect $1 \mathrm{~d}$ memory (Dubnau et al., 2003). Dopaminergic and octopaminergic neurons, which both innervate the $\mathrm{MB}$ lobes, are believed to carry the aversive and appetitive unconditioned stimulus reinforcement, respectively (Schwaerzel et al., 2003).

Correspondingly, learning and memory-associated cellular changes, collectively called memory traces, have been found in some of these structures at different stages of memory processing. In DPM neurons, odor-evoked $\mathrm{Ca}^{2+}$ response in the branch that innervates the $\mathrm{MB}$ vertical lobes ( $\alpha$ and $\alpha^{\prime}$ ) is enhanced $30 \mathrm{~min}$ after conditioning (Yu et al., 2005). In the MB, enhanced odor-evoked $\mathrm{Ca}^{2+}$ response is observed in the $\alpha$ lobe $24 \mathrm{~h}$ after spaced training (multiple training sessions with a rest between sessions), which produces LTM (Yu et al., 2006). In addition, a memory trace appearing as recruitment of new projection neurons (PNs) in the AL occurs immediately after conditioning but lasts only a few minutes, disappearing at $7 \mathrm{~min}$ after conditioning (Yu et al., 2004).

How these memory traces interact with each other is not yet clear. The memory trace in the AL PNs and that we observed in the $\mathrm{MB} \alpha^{\prime} / \beta^{\prime}$ neurons may appear in parallel after conditioning so STM may be formed in two independent places. The following observations support this possibility. One is that the memory trace in PNs is observed in the presynaptic specializations of PNs localized in the AL glomeruli (Yu et al., 2004). Second, we observed no change in the amount of odor-evoked $\mathrm{Ca}^{2+}$ response after olfactory training in the calyx, where synaptic connections are made between MB neurons and PNs. The spatial distribution of activity in the calyx was also not changed after olfactory training (data not shown). Therefore, the recruited new synaptic activities in the AL may not be transmitted to the MB to drive activity changes there at time points we studied.

The involvement of DPM neurons in memory consolidation relies on their projections to the axonal branches of the $\mathrm{MB} \alpha^{\prime} / \beta^{\prime}$ 
neurons (Keene et al., 2006). This suggests that memory consolidation may be performed by local interactions between DPM neurons and the MB $\alpha^{\prime} / \beta^{\prime}$ neurons, and the memory may reside in the $\alpha^{\prime} / \beta^{\prime}$ neurons during this process (Keene et al., 2006). This idea is also supported by the finding that the $\alpha^{\prime} / \beta^{\prime}$ neurons are required for memory acquisition and consolidation (Krashes et al., 2007). Our observation of a memory trace in the axonal branches of the $\mathrm{MB} \alpha^{\prime} / \beta^{\prime}$ neurons also provides a strong support to the idea that the memory initially resides in the $\alpha^{\prime} / \beta^{\prime}$ lobes. From this perspective, it is interesting that the memory trace in the DPM neurons is only observed in branches that innervate the MB vertical lobes (Yu et al., 2005). It is not known whether this memory trace is restricted further to branches that innervate the $\alpha^{\prime}$ lobe or is expressed in all branches that innervate vertical lobes. The memory trace in $\alpha^{\prime} / \beta^{\prime}$ neurons we observed here seems to appear before the memory trace in the DPM neurons, which was detected $\sim 30$ min after conditioning. Currently, we do not know whether there is any causal link between the two memory traces or whether they may exist independently. It should be interesting to see how the two memory traces are affected by manipulating activities of the DPM neurons and $\alpha^{\prime} / \beta^{\prime}$ neurons.

The LTM trace in the $\alpha$ lobe of the $\alpha / \beta$ neurons start to appear at $9 \mathrm{~h}$ after spaced training (Yu et al., 2006). It will be interesting to investigate whether the early memory trace we observed in the $\alpha^{\prime} / \beta^{\prime}$ neurons also exists after spaced training and how the STM trace is converted into the LTM trace. The LTM trace is blocked by mutation of the amnesiac gene (Yu et al., 2006), which is expressed in the DPM neurons (Waddell et al., 2000). Given the facts that the amnesiac mutation also blocks the intermediate memory trace in DPM neurons and that DPM projections to the axonal branches of the $\alpha^{\prime} / \beta^{\prime}$ neurons are sufficient for its role in memory consolidation, there is a very good possibility that the amnesiac mutation will disrupt the memory trace in the $\alpha^{\prime} / \beta^{\prime}$ neurons and this disruption results in the elimination of the LTM trace in the $\alpha / \beta$ neurons. Future studies addressing these issues should help us better understand how memory is formed and maintained in the brain.

\section{References}

Brand AH, Perrimon N (1993) Targeted gene expression as a means of altering cell fates and generating dominant phenotypes. Development 118:401-415.

Connolly JB, Roberts IJ, Armstrong JD, Kaiser K, Forte M, Tully T, O’Kane CJ (1996) Associative learning disrupted by impaired $\mathrm{G}_{\mathrm{s}}$ signaling in Drosophila mushroom bodies. Science 274:2104-2107.

Crittenden JR, Skoulakis EM, Han KA, Kalderon D, Davis RL (1998) Tripartite mushroom body architecture revealed by antigenic markers. Learn Mem 5:38-51.

Davis RL (2005) Olfactory memory formation in Drosophila: from molecular to systems neuroscience. Annu Rev Neurosci 28:275-302.

de Belle JS, Heisenberg M (1994) Associative odor learning in Drosophila abolished by chemical ablation of mushroom bodies. Science 263:692-695.

Dubnau J, Grady L, Kitamoto T, Tully T (2001) Disruption of neurotransmission in Drosophila mushroom body blocks retrieval but not acquisition of memory. Nature 411:476-480.

Dubnau J, Chiang AS, Grady L, Barditch J, Gossweiler S, McNeil J, Smith P, Buldoc F, Scott R, Certa U, Broger C, Tully T (2003) The staufen/pumilio pathway is involved in Drosophila long-term memory. Curr Biol 13:286-296.

Ferris J, Ge H, Liu L, Roman G (2006) G(o) signaling is required for Drosophila associative learning. Nat Neurosci 9:1036-1040.

Gerber B, Tanimoto H, Heisenberg M (2004) An engram found? Evaluating the evidence from fruit flies. Curr Opin Neurobiol 14:737-744.

Hallem EA, Carlson JR (2004) The odor coding system of Drosophila. Trends Genet 20:453-459.

Heisenberg M (2003) Mushroom body memoir: from maps to models. Nat Rev Neurosci 4:266-275.

Hildebrand JG, Shepherd GM (1997) Mechanisms of olfactory discrimination: converging evidence for common principles across phyla. Annu Rev Neurosci 20:595-631.

Isabel G, Pascual A, Preat T (2004) Exclusive consolidated memory phases in Drosophila. Science 304:1024-1027.

Kandel ER, Pittenger C (1999) The past, the future and the biology of memory storage. Philos Trans R Soc Lond B Biol Sci 354:2027-2052.

Keene AC, Stratmann M, Keller A, Perrat PN, Vosshall LB, Waddell S (2004) Diverse odor-conditioned memories require uniquely timed dorsal paired medial neuron output. Neuron 44:521-533.

Keene AC, Krashes MJ, Leung B, Bernard JA, Waddell S (2006) Drosophila dorsal paired medial neurons provide a general mechanism for memory consolidation. Curr Biol 16:1524-1530.

Kitamoto T (2001) Conditional modification of behavior in Drosophila by 

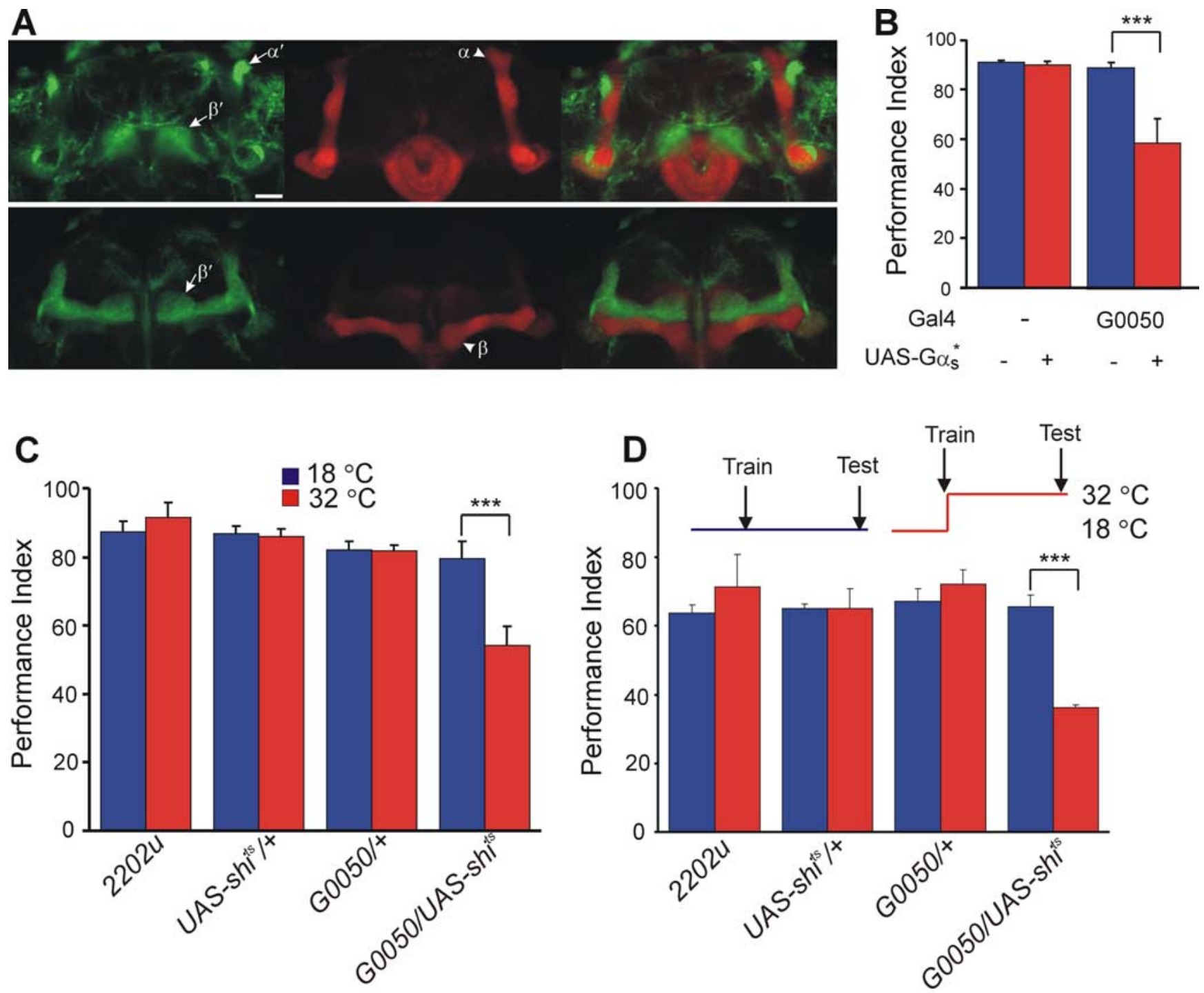

Figure 6. Disruption of the $G$-protein signaling in $\alpha^{\prime} / \beta^{\prime}$ neurons or blockade of their synaptic output impairs early memory. $A$, Green fluorescent protein (GFP) expression in the $\alpha^{\prime} / \beta^{\prime}$ lobes driven by the GAL 4 line G0050. First column, Two optical sections at the MB lobe region showing GFP expression. The arrows point to the $\alpha^{\prime} / \beta^{\prime}$ lobes. Second column, The same optical sections in the first column showing FASII counterstain that labels the $\alpha / \beta$ and $\gamma$ lobes in the MB (Crittenden et al., 1998). The arrowheads identify the $\alpha / \beta$ lobes. Third column, Merged images of the GFP fluorescence and FASIl staining. In the MB, G0050 drives GFP expression only in the $\alpha^{\prime} / \beta^{\prime}$ lobes. GFP expression is also seen outside the MB, but no expression is observed in the AL and central complex. Scale bar, $20 \mu \mathrm{m} . \boldsymbol{B}$, Impairment of immediate memory by disrupting the $\mathrm{G}$-protein signaling in MB neurons. Expression of constitutively active $G \alpha_{\mathrm{s}}^{*}$ only in $\alpha^{\prime} / \beta^{\prime}$ neurons impaired immediate $(3 \mathrm{~min})$ memory $\left({ }^{* * *} p<0.0001 ; n=5\right)$. C, Impairment of immediate ( $3 \mathrm{~min}$ ) memory by disrupting synaptic output from $\alpha^{\prime} / \beta^{\prime}$ neurons. At a restrictive temperature, expression of the temperature-sensitive Shi ${ }^{\text {ts }}$ only in $\alpha^{\prime} / \beta^{\prime}$ neurons impaired immediate memory ( $\left.{ }^{* * *} p<0.0001 ; n=5\right)$. D, Impairment of $1 \mathrm{~h}$ memory by disruption of synaptic output from $\alpha^{\prime} / \beta^{\prime}$ neurons. Flies were shifted to $32^{\circ} \mathrm{C}$ after training (red bars). In control groups, flies were kept at the permissive $18^{\circ} \mathrm{C}$ temperature (blue bars). Blocking synaptic output only in $\alpha^{\prime} / \beta^{\prime}$ neurons after training impaired $1 \mathrm{~h}$ memory $\left({ }^{* * *} p<0.0001 ; n=5\right)$. Error bars represent SEM.

targeted expression of a temperature-sensitive shibire allele in defined neurons. J Neurobiol 47:81-92.

Krashes MJ, Keene AC, Leung B, Armstrong JD, Waddell S (2007) Sequential use of mushroom body neuron subsets during Drosophila odor memory processing. Neuron 53:103-115.

Lee T, Lee A, Luo L (1999) Development of the Drosophila mushroom bodies: sequential generation of three distinct types of neurons from a neuroblast. Development 126:4065-4076.

Lin HH, Lai JS, Chin AL, Chen YC, Chiang AS (2007) A map of olfactory representation in the Drosophila mushroom body. Cell 128:1205-1217.

Malinow R, Malenka RC (2002) AMPA receptor trafficking and synaptic plasticity. Annu Rev Neurosci 25:103-126.

McGuire SE, Le PT, Davis RL (2001) The role of Drosophila mushroom body signaling in olfactory memory. Science 293:1330-1333.

McGuire SE, Le PT, Osborn AJ, Matsumoto K, Davis RL (2003) Spatiotemporal rescue of memory dysfunction in Drosophila. Science 302:1765-1768.

Pascual A, Preat T (2001) Localization of long-term memory within the Drosophila mushroom body. Science 294:1115-1117.
Perez-Orive J, Mazor O, Turner GC, Cassenaer S, Wilson RI, Laurent G (2002) Oscillations and sparsening of odor representations in the mushroom body. Science 297:359-365.

Reiff DF, Ihring A, Guerrero G, Isacoff EY, Joesch M, Nakai J, Borst A (2005) In vivo performance of genetically encoded indicators of neural activity in flies. J Neurosci 25:4766-4778.

Schwaerzel M, Heisenberg M, Zars T (2002) Extinction antagonizes olfactory memory at the subcellular level. Neuron 35:951-960.

Stevens CF, Sullivan JM (1998) Regulation of the readily releasable vesicle pool by protein kinase C. Neuron 21:885-893.

Thompson RF (2005) In search of memory traces. Annu Rev Psychol 56:1-23.

Tully T, Quinn WG (1985) Classical conditioning and retention in normal and mutant Drosophila melanogaster. J Comp Physiol A Neuroethol Sens Neural Behav Physiol 157:263-277.

Tully T, Preat T, Boynton SC, Del Vecchio M (1994) Genetic dissection of consolidated memory in Drosophila. Cell 79:35-47.

Turner GC, Bazhenov M, Laurent G (2008) Olfactory representations by Drosophila mushroom body neurons. J Neurophysiol 99:734-746. 
Waddell S, Armstrong JD, Kitamoto T, Kaiser K, Quinn WG (2000) The amnesiac gene product is expressed in two neurons in the Drosophila brain that are critical for memory. Cell 103:805-813.

Wang JW, Wong AM, Flores J, Vosshall LB, Axel R (2003) Two-photon calcium imaging reveals an odor-evoked map of activity in the fly brain. Cell 112:271-282.

Wang Y, Wright NJ, Guo H, Xie Z, Svoboda K, Malinow R, Smith DP, Zhong Y (2001) Genetic manipulation of the odor-evoked distributed neural activity in the Drosophila mushroom body. Neuron 29:267-276.

Wang Y, Guo HF, Pologruto TA, Hannan F, Hakker I, Svoboda K, Zhong Y (2004) Stereotyped odor-evoked activity in the mushroom body of Drosophila revealed by green fluorescent protein-based $\mathrm{Ca}^{2+}$ imaging. J Neurosci 24:6507-6514.
Xia S, Miyashita T, Fu TF, Lin WY, Wu CL, Pyzocha L, Lin IR, Saitoe M, Tully T, Chiang AS (2005) NMDA receptors mediate olfactory learning and memory in Drosophila. Curr Biol 15:603-615.

Yu D, Ponomarev A, Davis RL (2004) Altered representation of the spatial code for odors after olfactory classical conditioning; memory trace formation by synaptic recruitment. Neuron 42:437-449.

Yu D, Keene AC, Srivatsan A, Waddell S, Davis RL (2005) Drosophila DPM neurons form a delayed and branch-specific memory trace after olfactory classical conditioning. Cell 123:945-957.

Yu D, Akalal DB, Davis RL (2006) Drosophila alpha/beta mushroom body neurons form a branch-specific, long-term cellular memory trace after spaced olfactory conditioning. Neuron 52:845-855.

Zars T, Fischer M, Schulz R, Heisenberg M (2000) Localization of a shortterm memory in Drosophila. Science 288:672-675. 